sciendo

\section{TATRA \\ MOUNTaiNS \\ Mathematical Publications}

DOI: $10.2478 / \mathrm{tmmp}-2020-0032$

Tatra Mt. Math. Publ. 77 (2020), 53-58

\title{
A NOTE ON EXPANSIONS OF RATIONAL NUMBERS BY CERTAIN SERIES
}

\author{
Symon SERBEnyUK \\ Vinnytsia, UKRAINE
}

\begin{abstract}
This paper deals with representations of rational numbers defined in terms of numeral systems that are certain generalizations of the classical $q$-ary numeral system.
\end{abstract}

In [1], expansions of the following form of real numbers from the interval $[0,1]$ were considered:

$$
\frac{\varepsilon_{1}}{q_{1}}+\frac{\varepsilon_{2}}{q_{1} q_{2}}+\cdots+\frac{\varepsilon_{k}}{q_{1} q_{2} \ldots q_{k}}+\cdots .
$$

Here $Q \equiv\left(q_{k}\right)$ is a fixed sequence of positive integers, $q_{k}>1$, and $\left(\Theta_{k}\right)$ is a sequence of the sets $\Theta_{k} \equiv\left\{0,1, \ldots, q_{k}-1\right\}$, as well as $\varepsilon_{k} \in \Theta_{k}$.

Note that the last-mentioned expansion under the condition $q_{k}=$ const $=q$ for all positive integers $k$, where $1<q \in \mathbb{N}$ ( $\mathbb{N}$ is the set of all positive integers), is the q-ary expansion of real numbers from $[0,1]$, i.e.,

$$
\frac{\varepsilon_{1}}{q}+\frac{\varepsilon_{2}}{q^{2}}+\cdots+\frac{\varepsilon_{k}}{q^{k}}+\cdots,
$$

where $\varepsilon_{k} \in\{0,1, \ldots, q-1\}$. In this case, a number is a rational number if and only if a sequence $\left(\varepsilon_{k}\right)$ is periodic.

However, the problem of the rationality/irrationality of numbers defined in terms of generalizations of the q-ary numeral system is difficult. A version of this problem for expansions of form (1) was introduced in the paper [1] and has been studied by a number of researchers. For example, G. Cantor, P. A. Diananda, A. Oppenheim, P. Erdős, J. Hančl, E. G. Straus, P. Rucki, P. Kuhapatanakul, V. Laohakosol and other scientists studied this problem (see references). Known results include different conditions satisfied by $\left(\varepsilon_{k}\right)$ and/or $\left(q_{k}\right)$.

(C) 2020 Mathematical Institute, Slovak Academy of Sciences. 2010 Mathematics Subject Classification: 11K55, 11J72.

Keywords: expansions of real numbers, rational number, q-ary numeral system.

Licensed under the Creative Commons Attribution-NC-ND4.0 International Public License. 


\section{SYMON SERBENYUK}

In this paper, the main attention is given to calculating values of $\varepsilon_{k}$ for an arbitrary rational number and a fixed sequence $\left(q_{k}\right)$. That is, the problem of the rationality is investigated for the case of a numeral system whose base is an infinite sequence of positive integers.

By $x=\Delta_{\varepsilon_{1} \varepsilon_{2} \ldots \varepsilon_{k} \ldots}^{Q}$ denote a number $x \in[0,1]$ represented by series (1). Suppose $c_{1}, c_{2}, \ldots, c_{m}$ is an ordered tuple of integers such that $c_{i} \in\left\{0,1, \ldots, q_{i}-1\right\}$ for $i=\overline{1, m}$. A cylinder $\Delta_{c_{1} c_{2} \ldots c_{m}}^{Q}$ of rank $m$ with base $c_{1} c_{2} \ldots c_{m}$ is a set of the form

$$
\Delta_{c_{1} c_{2} \ldots c_{m}}^{Q} \equiv\left\{x: x=\Delta_{c_{1} c_{2} \ldots c_{m} \varepsilon_{m+1} \varepsilon_{m+2} \ldots \varepsilon_{m+k} \ldots}^{Q}\right\} .
$$

That is, any cylinder $\Delta_{c_{1} c_{2} \ldots c_{m}}^{Q}$ is a closed interval of the form

$$
\left[\Delta_{c_{1} c_{2} \ldots c_{m} 000 \ldots,}^{Q}, \Delta_{c_{1} c_{2} \ldots c_{m}\left[q_{m+1}-1\right]\left[q_{m+2}-1\right]\left[q_{m+3}-1\right] \ldots}^{Q}\right] .
$$

Define the shift operator $\sigma$ of expansion (1) as

It is easy to see that

$$
\sigma(x)=\sigma\left(\Delta_{\varepsilon_{1} \varepsilon_{2} \ldots \varepsilon_{k} \ldots}^{Q}\right)=\sum_{k=2}^{\infty} \frac{\varepsilon_{k}}{q_{2} q_{3} \ldots q_{k}}=q_{1} \Delta_{0 \varepsilon_{2} \ldots \varepsilon_{k} \ldots}^{Q} .
$$

$$
\begin{aligned}
\sigma^{n}(x) & =\sigma^{n}\left(\Delta_{\varepsilon_{1} \varepsilon_{2} \ldots \varepsilon_{k} \ldots}^{Q}\right) \\
& =\sum_{k=n+1}^{\infty} \frac{\varepsilon_{k}}{q_{n+1} q_{n+2} \ldots q_{k}}=q_{1} \ldots q_{n} \Delta_{\underbrace{Q \ldots 0}_{n}}^{\underbrace{}_{n} \ldots \varepsilon_{n+1} \varepsilon_{n+2} \ldots} .
\end{aligned}
$$

Therefore,

$$
x=\sum_{i=1}^{n} \frac{\varepsilon_{i}}{q_{1} q_{2} \ldots q_{i}}+\frac{1}{q_{1} q_{2} \ldots q_{n}} \sigma^{n}(x) .
$$

Lemma 1. Let $x \in(0,1)$ be a rational number represented by series (11). If $x=\frac{p}{r}=\Delta_{\varepsilon_{1} \varepsilon_{2} \ldots \varepsilon_{n} \ldots}^{Q}$, then the equality

holds for all $n \in \mathbb{N}$, where

$$
\varepsilon_{n}=\left[\frac{\Delta_{n}}{r}\right]
$$

$$
\Delta_{n}=p q_{1} q_{2} \cdots q_{n}-r\left(\varepsilon_{1} q_{2} q_{3} \cdots q_{n}+\varepsilon_{2} q_{3} q_{4} \cdots q_{n}+\cdots+\varepsilon_{n-1} q_{n}\right) .
$$

P r o of. Let $\frac{p}{r}$ be a fixed number, where $(p, r)=1, p<r$, and $p \in \mathbb{N}, r \in \mathbb{N}$. Then

$$
\frac{p}{r}=\sum_{n=1}^{\infty} \frac{\varepsilon_{n}}{q_{1} q_{2} \cdots q_{n}} .
$$

Remark 1. Note that, since $x \in \Delta_{c_{1} c_{2} \ldots c_{m}}^{Q}$ but

$$
\Delta_{c_{1} c_{2} \ldots c_{m}}^{Q}\left[q_{m+1}-1\right]\left[q_{m+2}-1\right] \ldots=\Delta_{c_{1} c_{2} \ldots c_{m-1}\left[c_{m}+1\right] 000 \ldots}^{Q},
$$

we assume that

$$
\Delta_{c_{1} c_{2} \ldots c_{m-1}\left[c_{m}\right] 000 \ldots}^{Q} \leq x<\Delta_{c_{1} c_{2} \ldots c_{m-1}\left[c_{m}+1\right] 000 \ldots}^{Q}
$$


Let us prove this lemma. It is easy to see that

That is

$$
\frac{p}{r} \in \Delta_{\varepsilon_{1}}^{Q}=\left[\Delta_{\varepsilon_{1} 000 \ldots}^{Q}, \Delta_{\varepsilon_{1}\left[q_{2}-1\right]\left[q_{3}-1\right] \ldots}^{Q}\right]=\left[\frac{\varepsilon_{1}}{q_{1}}, \frac{\varepsilon_{1}+1}{q_{1}}\right] .
$$

$$
\frac{\varepsilon_{1}}{q_{1}} \leq \frac{p}{r}<\frac{\varepsilon_{1}+1}{q_{1}}, \quad \varepsilon_{1} r \leq p q_{1}<r\left(\varepsilon_{1}+1\right), \quad \varepsilon_{1} \leq \frac{p q_{1}}{r}<\varepsilon_{1}+1 .
$$

So,

$$
\varepsilon_{1}=\left[\frac{p}{r} q_{1}\right], \quad \text { where }[x] \text { is the integer part of } x .
$$

Now we get

Whence,

$$
\frac{p}{r} \in \Delta_{\varepsilon_{1} \varepsilon_{2}}^{Q}=\left[\frac{q_{2} \varepsilon_{1}+\varepsilon_{2}}{q_{1} q_{2}}, \frac{q_{2} \varepsilon_{1}+\varepsilon_{2}+1}{q_{1} q_{2}}\right] .
$$

$$
\frac{q_{2} \varepsilon_{1}+\varepsilon_{2}}{q_{1} q_{2}} \leq \frac{p}{r}<\frac{q_{2} \varepsilon_{1}+\varepsilon_{2}+1}{q_{1} q_{2}}, \quad \varepsilon_{2} \leq \frac{p q_{1} q_{2}-r q_{2} \varepsilon_{1}}{r}<\varepsilon_{2}+1 .
$$

So,

$$
\varepsilon_{2}=\left[\frac{p q_{1} q_{2}-r q_{2} \varepsilon_{1}}{r}\right] .
$$

In the third step, we have

and

$$
\frac{p}{r} \in \Delta_{\varepsilon_{1} \varepsilon_{2} \varepsilon_{3}}^{Q}=\left[\frac{\varepsilon_{1} q_{2} q_{3}+\varepsilon_{2} q_{3}+\varepsilon_{3}}{q_{1} q_{2} q_{3}}, \frac{\varepsilon_{1} q_{2} q_{3}+\varepsilon_{2} q_{3}+\varepsilon_{3}+1}{q_{1} q_{2} q_{3}}\right]
$$

$$
\varepsilon_{3}=\left[\frac{p q_{1} q_{2} q_{3}-r\left(\varepsilon_{1} q_{2} q_{3}+\varepsilon_{2} q_{3}\right)}{r}\right] .
$$

In the $n$-th step, we obtain

$$
\begin{aligned}
& \frac{p}{r} \in \Delta_{\varepsilon_{1} \varepsilon_{2} \ldots \varepsilon_{n-1} \varepsilon_{n}}^{Q}=\left[\sum_{i=1}^{n-1} \frac{\varepsilon_{i}}{q_{1} q_{2} \cdots q_{i}}+\frac{\varepsilon_{n}}{q_{1} q_{2} \cdots q_{n}}, \sum_{i=1}^{n-1} \frac{\varepsilon_{i}}{q_{1} q_{2} \cdots q_{i}}+\frac{\varepsilon_{n}+1}{q_{1} q_{2} \cdots q_{n}}\right] \\
&=\left[\frac{\varepsilon_{1} q_{2} q_{3} \cdots q_{n}+\varepsilon_{2} q_{3} q_{4} \cdots q_{n}+\cdots+\varepsilon_{n-1} q_{n}+\varepsilon_{n}}{q_{1} q_{2} \cdots q_{n}},\right. \\
&\left.\frac{\varepsilon_{1} q_{2} q_{3} \cdots q_{n}+\cdots+\varepsilon_{n-1} q_{n}+\varepsilon_{n}+1}{q_{1} q_{2} \cdots q_{n}}\right] .
\end{aligned}
$$

Let $\varsigma_{n}$ denote the sum

$$
\varepsilon_{1} q_{2} q_{3} \cdots q_{n}+\varepsilon_{2} q_{3} q_{4} \cdots q_{n}+\cdots+\varepsilon_{n-1} q_{n} .
$$

Then

$$
\frac{\varsigma_{n}+\varepsilon_{n}}{q_{1} q_{2} \cdots q_{n}} \leq \frac{p}{r}<\frac{\varsigma_{n}+\varepsilon_{n}+1}{q_{1} q_{2} \cdots q_{n}}, \quad \varepsilon_{n} \leq \frac{p q_{1} q_{2} \cdots q_{n}-r \varsigma_{n}}{r}<\varepsilon_{n}+1 .
$$

This completes the proof. 
Denoting by $\Delta_{n}=p q_{1} q_{2} \cdots q_{n}-r \varsigma_{n}$, we get

$$
\varepsilon_{n}=\left[\frac{\Delta_{n}}{r}\right] \text {. }
$$

Also, for $n \geq 2$ the condition $\varsigma_{n}=\varsigma_{n-1} q_{n}+\varepsilon_{n-1} q_{n}$ holds and

$$
\Delta_{n}=q_{n}\left(\Delta_{n-1}-r \varepsilon_{n-1}\right) \text {. }
$$

So, the following statement is true.

Lemma 2. Let $x \in(0,1)$ be a rational number represented by series (11). If $x=\frac{p}{r}=\Delta_{\varepsilon_{1} \varepsilon_{2} \ldots \varepsilon_{n} \ldots}^{Q}$, then the equality

$$
\varepsilon_{n}=\left[\frac{q_{n}\left(\Delta_{n-1}-r \varepsilon_{n-1}\right)}{r}\right]
$$

holds for all $1<n \in \mathbb{N}$, where $\Delta_{1}=p q_{1}$ and $\varepsilon_{1}=\left[\frac{\Delta_{1}}{r}\right]$.

Theorem. A number $x=\Delta_{\varepsilon_{1} \varepsilon_{2} \ldots \varepsilon_{n} \ldots}^{Q} \in(0,1)$ is a rational number $\frac{p}{r}$, where $p, r \in \mathbb{N},(p, r)=1$, and $p<r$, if and only if the condition

$$
\varepsilon_{n}=\left[\frac{q_{n}\left(\Delta_{n-1}-r \varepsilon_{n-1}\right)}{r}\right]
$$

holds for all $1<n \in \mathbb{N}$, where $\Delta_{1}=p q_{1}, \varepsilon_{1}=\left[\frac{\Delta_{1}}{r}\right]$, and $[a]$ is the integer part of $a$.

P r o of. The necessity follows from previous two lemmas.

Let us prove that the sufficiency is true. Suppose that the following sequence of conditions is true:

$$
\begin{aligned}
& \varepsilon_{1}=\left[\frac{p}{r} q_{1}\right], \quad \varepsilon_{2}=\left[\frac{p q_{1} q_{2}-r q_{2} \varepsilon_{1}}{r}\right], \quad \varepsilon_{3}=\left[\frac{p q_{1} q_{2} q_{3}-r\left(\varepsilon_{1} q_{2} q_{3}+\varepsilon_{2} q_{3}\right)}{r}\right], \ldots \\
& \varepsilon_{n}=\left[\frac{p q_{1} q_{2} \cdots q_{n}}{r}-\left(\varepsilon_{1} q_{2} q_{3} \cdots q_{n}+\varepsilon_{2} q_{3} q_{4} \cdots q_{n}+\cdots+\varepsilon_{n-1} q_{n}\right)\right]=\left[\frac{\Delta_{n}}{r}\right], \ldots
\end{aligned}
$$

It follows from equality (2) that

$$
\begin{gathered}
x=\frac{p}{r}=\frac{\varepsilon_{1} q_{2} q_{3} \cdots q_{n}+\varepsilon_{2} q_{3} q_{4} \cdots q_{n}+\cdots+\varepsilon_{n-1} q_{n}+\varepsilon_{n}}{q_{1} q_{2} \cdots q_{n}}+\frac{\sigma^{n}\left(\frac{p}{r}\right)}{q_{1} q_{2} \cdots q_{n}}, \\
\sigma^{n}\left(\frac{p}{r}\right)=\frac{\Delta_{n}-r \varepsilon_{n}}{r}=\frac{\Delta_{n}}{r}-\varepsilon_{n},
\end{gathered}
$$

and

$$
\varepsilon_{n}=\frac{\Delta_{n}}{r}-\sigma^{n}\left(\frac{p}{r}\right) .
$$

It follows from the last-mentioned relationship and relationship (3) that

$$
0 \leq \varepsilon_{n}=\left[\frac{\Delta_{n}}{r}\right]=\frac{\Delta_{n}}{r}-\sigma^{n}\left(\frac{p}{r}\right)=\left[\frac{\Delta_{n}}{r}-\sigma^{n}\left(\frac{p}{r}\right)\right] .
$$


That is,

$$
\sigma^{n}\left(\frac{p}{r}\right)=\left\{\frac{\Delta_{n}}{r}\right\},
$$

where $\{a\}$ is the fractional part of $a$.

Remark 2. Clearly,

$$
0 \leq \sigma^{n}(x) \leq 1
$$

for an arbitrary $x \in[0,1]$. However, for any number of the form

$$
\begin{aligned}
x=x_{1} & =\Delta_{\varepsilon_{1} \varepsilon_{2} \ldots \varepsilon_{n-1} \varepsilon_{n} 000 \ldots}^{Q} \\
& =\Delta_{\varepsilon_{1} \varepsilon_{2} \ldots \varepsilon_{n-1}\left[\varepsilon_{n}-1\right]\left[q_{n+1}-1\right]\left[q_{n+2}-1\right]\left[q_{n+3}-1\right] \ldots}^{Q}=x_{2}
\end{aligned}
$$

the following conditions hold:

$$
\begin{aligned}
\sigma^{n}(x) & =\sigma^{n}\left(\Delta_{\varepsilon_{1} \varepsilon_{2} \ldots \varepsilon_{n-1} \varepsilon_{n} 000 \ldots}^{Q}\right) \\
& =\sum_{k=n+1}^{\infty} \frac{0}{q_{n+1} q_{n+2} \cdots q_{k}}=0, \\
\sigma^{n}(x) & =\sigma^{n}\left(\Delta_{\varepsilon_{1} \varepsilon_{2} \ldots \varepsilon_{n-1}\left[\varepsilon_{n}-1\right]\left[q_{n+1}-1\right]\left[q_{n+2}-1\right]\left[q_{n+3}-1\right] \ldots}^{Q}\right) \\
& =\sum_{k=n+1}^{\infty} \frac{q_{k}-1}{q_{n+1} q_{n+2} \cdots q_{k}}=1 .
\end{aligned}
$$

Since the condition $\sigma^{n}\left(x_{2}\right)=1$ holds, we can use only the first representation of number $x$, i.e., the form $x_{1}$, and for these numbers the condition

$$
\sigma^{n}(x)=\sigma^{n}\left(x_{1}\right)=0 \quad \text { holds. }
$$

In addition, note that

$$
\varsigma_{n}=\frac{p q_{1} q_{2} \cdots q_{n}-\Delta_{n}}{r} .
$$

Whence for an arbitrary $n \in \mathbb{N}$

$$
\begin{aligned}
x & =\sum_{k=1}^{n} \frac{\varepsilon_{k}}{q_{1} q_{2} \cdots q_{k}}+\frac{\sigma^{n}(x)}{q_{1} q_{2} \cdots q_{n}} \\
& =\frac{\varepsilon_{1} q_{2} q_{3} \cdots q_{n}+\varepsilon_{2} q_{3} q_{4} \cdots q_{n}+\cdots+\varepsilon_{n-1} q_{n}+\varepsilon_{n}}{q_{1} q_{2} \cdots q_{n}}+\frac{\sigma^{n}(x)}{q_{1} q_{2} \cdots q_{n}} \\
& =\frac{\varsigma_{n}+\varepsilon_{n}}{q_{1} q_{2} \cdots q_{n}}+\frac{\left\{\frac{\Delta_{n}}{r}\right\}}{q_{1} q_{2} \cdots q_{n}}=\frac{\frac{p q_{1} q_{2} \cdots q_{n}-\Delta_{n}}{r}+\varepsilon_{n}}{q_{1} q_{2} \cdots q_{n}}+\frac{\frac{\Delta_{n}}{r}-\left[\frac{\Delta_{n}}{r}\right]}{q_{1} q_{2} \cdots q_{n}} \\
& =\frac{p q_{1} q_{2} \cdots q_{n}-\Delta_{n}+r \varepsilon_{n}}{r q_{1} q_{2} \cdots q_{n}}+\frac{\frac{\Delta_{n}}{r}-\varepsilon_{n}}{q_{1} q_{2} \cdots q_{n}}=\frac{p}{r} .
\end{aligned}
$$

This completes the proof. 


\section{SYMON SERBENYUK}

Let us consider certain examples. Suppose

Then

$$
x=\Delta_{\varepsilon_{1} \varepsilon_{2} \ldots \varepsilon_{n} \ldots}^{(2 n+1)}=\sum_{n=1}^{\infty} \frac{\varepsilon_{n}}{3 \cdot 5 \cdot 7 \cdots(2 n+1)} .
$$

$$
\frac{1}{4}=\Delta_{035229[11] 4 \ldots}^{(2 n+1)}, \quad \frac{3}{8}=\Delta_{104341967 \ldots}^{(2 n+1)} .
$$

\section{REFERENCES}

[1] CANTOR, G.: Ueber die einfachen Zahlensysteme, Z. Math. Phys. 14 (1869), 121-128. (In German)

[2] DIANANDA, P. H.-OPPENHEIM, A.: Criteria for irrationality of certain classes of numbers II, Amer. Math. Monthly 62 (1955), no. 4, 222-225.

[3] ERDŐS, P.-STRAUS, E. G.: On the irrationality of certain series, Pacific J. Math. 55 (1974), no. 1, 85-92.

[4] HANČL, J.: A note to the rationality of infinite series I, Acta Math. Inf. Univ. Ostrav. 5 (1997), no. 1, 5-11.

[5] HANČL, J.-RUCKI, P.: A note to the transcendence of special infinite series, Math. Slovaca 56 (2006), no. 4, 409-414.

[6] KUHAPATANAKUL, P.-LAOHAKOSOL, V.: Irrationality of some series with rational terms, Kasetsart J. (Nat. Sci.) 35 (2001), 205-209.

[7] OPPENHEIM, A.: Criteria for irrationality of certain classes of numbers, Amer. Math. Monthly 61 (1954), no. 4, 235-241.

Received September 18, 2019

45 Shchukina St. 21012-Vinnytsia UKRAINE

E-mail: simon6@ukr.net 